Article

\title{
Oxide-Free Bonding of III-V-Based Material on Silicon and Nano-Structuration of the Hybrid Waveguide for Advanced Optical Functions
}

Konstantinos Pantzas ${ }^{1}$, Ahmad Itawi ${ }^{1}$, Isabelle Sagnes ${ }^{1}$, Gilles Patriarche ${ }^{1}$, Eric Le Bourhis ${ }^{\text {2, }}$ Anatole Lupu ${ }^{3}$, Henri Benisty ${ }^{4}$ and Anne Talneau ${ }^{1, *}$

1 Laboratoire de Photonique et de Nanostructures, route de Nozay, F-91460 Marcoussis, France; E-Mails: konstantinos.pantzas@lpn.cnrs.fr (K.P.); ahmad.itawi@lpn.cnrs.fr (A.I.); isabelle.sagnes@1pn.cnrs.fr (I.S.); gilles.patriarche@lpn.cnrs.fr (G.P.)

2 Institut P', CNRS Université de Poitiers-Bd Marie Pierre Cuire, B.P. 30179, 86962 Futuroscope-Chasseneuil Cedex, France; E-Mail: eric.le.bourhis@univ-poitiers.fr

3 Institut d'Electronique Fondamentale, Université d'Orsay, 91405 Orsay, France; E-Mail: Anatole.lupu@ief.u-psud.fr

4 Laboratoire Charles Fabry, UMR 8501, Institut d'Optique, CNRS, Univ Paris Sud 11, 2 Avenue Augustin Fresnel, 91127 Palaiseau Cedex, France; E-Mail: henri.benisty@ institutoptique.fr

* Author to whom correspondence should be addressed; E-Mail: anne.talneau@lpn.cnrs.fr Tel.: +33-169-636-146.

Received: 30 September 2015 / Accepted: 26 October 2015 / Published: 29 October 2015

\begin{abstract}
Oxide-free bonding of III-V-based materials for integrated optics is demonstrated on both planar Silicon (Si) surfaces and nanostructured ones, using Silicon on Isolator (SOI) or Si substrates. The hybrid interface is characterized electrically and mechanically. A hybrid InP-on-SOI waveguide, including a bi-periodic nano structuration of the silicon guiding layer is demonstrated to provide wavelength selective transmission. Such an oxide-free interface associated with the nanostructured design of the guiding geometry has great potential for both electrical and optical operation of improved hybrid devices.
\end{abstract}

Keywords: silicon photonics hybrid integration; III-V materials; wafer bonding; oxide-free bonding; silicon waveguide nanopatterning; telecom optical functions 


\section{Introduction}

The future of all optical networks links relies upon hybrid silicon photonics. However, silicon is not able to produce two essential optical functions: photon emission and optical isolation. Bonding is then the best approach to take advantage of materials providing these optical functionalities: with adequate design of a guiding stack, bonded III-V semiconductors then provide light amplification and emission, almost inexistent in silicon, and bonded garnets provide optical isolation $[1,2]$.

Hybrid bonding of III-V on Si has been intensively developed for integrated photonics on silicon, but mainly with the help of an intermediate layer that provides bonding at annealing temperatures in the 250-300 ${ }^{\circ} \mathrm{C}$ range compatible with CMOS processing. This bonding approach has indeed produced sophisticated integrated devices [3,4], based on classical photonic integrated circuit schemes, taking advantage of the $\mathrm{Si}$ guiding layer to route optical signals as well. The intermediate bonding layer is usually a dielectric material $\left(\mathrm{SiO}_{2}, \mathrm{BCB}\right)$, with a typical thickness in the 50-100 nm range [5-7] or a very thin $\sim 5-10 \mathrm{~nm} \mathrm{SiO}_{2}$ layer [8,9]. For integrated optoelectronic devices, this dielectric material layer unfortunately precludes electrical injection across the interface: carrier injection is then performed through the III-V-doped layers, and thus along the n-doped InP layer which is very thin. As a negative consequence, any intense carrier flow produces a large Joule overheating, hampering high output power of, e.g., hybrid lasers through a large thermal roll-off $[5,8]$.

Oxide-free bonding has been scarcely investigated. Electrical operation across the hybrid interface potentially provides immense gains in terms of thermal-budget improvement, chip power consumption, and integration of driving electronics, welcome to boost data transfer rates. Bonding without an intentional additional layer has been obtained in Arakawa's group [10,11], allowing electrical operation under carrier injection through the interface. Nevertheless, in this work, a thin oxide layer is generated during bonding processes.

When both materials are associated with a sufficiently thin intermediate layer in channel waveguiding structures, the bottom III-V surface is flat, so the optical guided mode profile is mainly determined by the waveguide shape patterned in the silicon guiding layer, usually a rib waveguide [5,6]. Implementation of an advanced optical function requires additional processing steps to produce the structuration properly ensuring the intended operation, e.g., for wavelength selective lasing operation, a grating on top of the guiding layer [12], or a lateral grating [13].

Based on existing expertise on heteroepitaxial bonding of GaAs on InP [14], we have developed heteroepitaxial bonding of III-V materials on Si and SOI substrates for telecom wavelengths. This oxide-free bonding technique has demonstrated atomic-plane-thick reconstruction across the interface, leading to a high bonding energy and no degradation of the photoluminescence of bonded quantum wells located close to the hybrid interface $[15,16]$.

We start here by demonstrating that such a hetero interface can be electrically operated at sizable current densities, paving the way to electrical injection of hybrid devices through the substrate.

Next, for photonic purposes, we implement a uniquely designed sub-wavelength silicon waveguide patterning that provides in a single technological step advanced optical functions while keeping the Si guiding surface planar enough for maintaining the oxide-free bonding compatibility. For this photonic study, we first demonstrate through mechanical characterization of the interface that bona fide oxide-free bonding is obtained on such a nanopatterned waveguide. Secondly, we explain the design of a sub- $\lambda 2 \mathrm{D}$ 
photonic bandgap structuration in the lateral claddings of the silicon waveguide that features a large versatility for tailoring the spatial as well as the spectral characteristics of the guided mode, be it in an intended passive or active guide section. We show here on the example of an hybrid InP on SOI waveguide that, according to this design, a sub- wavelength patterned Si guiding layer on SOI including a bi-periodic nanostructuration properly performs its intended wavelength selective operation of the hybrid waveguide. Such a design opens the road towards monomode oxide-free hybrid lasers.

\section{Oxide-Free Bonding of III-V Material on Planar and Patterned Silicon: Experiment and Characterizations}

We start by a brief account of the fabrication and bonding processes.

Heteroepitaxial bonding of InP on $\mathrm{Si}$ or $\mathrm{SOI}$ is performed at die size $\left(\sim 1 \mathrm{~cm}^{2}\right)$. Both surfaces are prepared in an oxide-free manner [17,18], and annealed at $500{ }^{\circ} \mathrm{C}$ during $90 \mathrm{~min}$. $\mathrm{No}_{2}$ out gassing is visible, the $\mathrm{H}_{2}$ by-products having the ability to easily diffuse within both crystalline lattices. This was also the case when oxide-free bonding was performed for III-V on III-V in order to associate GaAs/AlGaAs Bragg mirrors on InP-based cavities for $1.55 \mu \mathrm{m}$ Vertical Cavity Surface Emitting Lasers (VCSEL) [14-19].

The III-V bonded membrane composition and thickness are tailored according to the characterization pursued. All characterizations are performed after removing the InP substrate and the InGaAs stop etch layer by selective chemical etching.

\subsection{Electrical Characterization}

For electrical studies, the $\mathrm{n}-\mathrm{InP} / \mathrm{n}$-Si isotype heterojunctions were investigated both theoretically and experimentally. The band diagram of the heterojunction was computed numerically using the PC1D software, which relies on Anderson's rule for the computation of band offsets [20]. The electron concentration is taken to be $3.0 \times 10^{18} \mathrm{~cm}^{-3}$ in n-type $\mathrm{InP}$, and $1.0 \times 10^{19} \mathrm{~cm}^{-3}$ in n-type $\mathrm{Si}$.

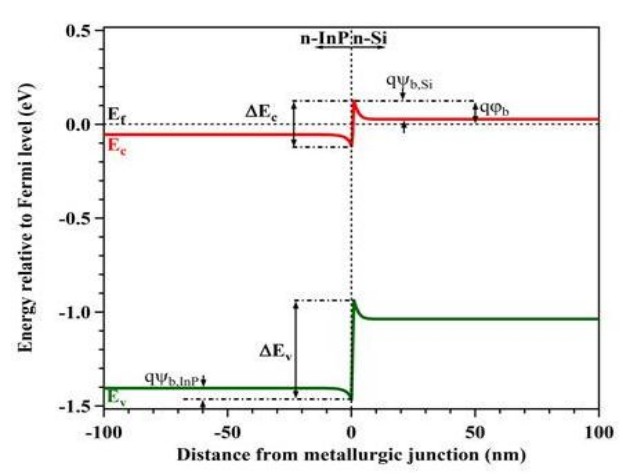

(a)

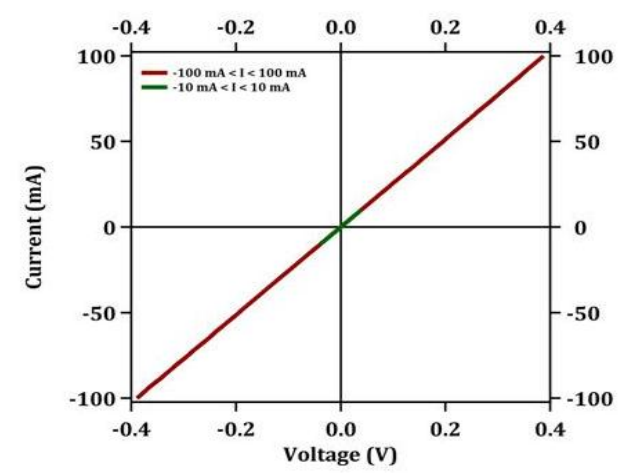

(b)

Figure 1. (a) Computed band diagram of the n-InP/n-Si heterojunction, (b) 4-points measurement of the $\mathrm{I}(\mathrm{V})$ characteristic of the hybrid interface.

Figure 1a shows the computed band diagram. The simulations reveal that the conduction and valence band offsets are $241 \mathrm{meV}$ and $527 \mathrm{meV}$, respectively. While the valence band offset is relatively large, this is not expected to adversely affect current transport in the diode, as this is a majority carrier device [20]. 
Voltage drops of $60.5 \mathrm{mV}$ and $98.5 \mathrm{mV}$ are expected to occur in the conduction band in $\mathrm{InP}$ and $\mathrm{Si}$, respectively, giving a total built-in voltage drop of $159 \mathrm{mV}$. Finally, the expected barrier height that electrons must overcome for charge flow to occur is estimated at $126 \mathrm{mV}$.

The modelled diode was fabricated experimentally. The doped InP membrane was grown on an InGaAs etch-stop layer on an InP substrate using metal-organic vapor phase epitaxy. The grown sample was then bonded to n-doped Si using the procedure described in [15]. The membrane was selectively separated from the InP substrate, and $500 \mu \mathrm{m}$ wide square mesa were etched in InP down to Si by a $\mathrm{Cl}_{2}-\mathrm{H}_{2}$-based Inductively-Coupled-Plasma Reactive-Ion-Etching (ICP-RIE) process. Contact pads with two concentric ring pairs were then defined using lithography and lift-off. The pair of Si contacts were formed by $\mathrm{Al}$ deposition, $\mathrm{HF}$ de-oxidation was performed prior to metal deposition. The pair of InP contacts were defined during a second lithography step, formed by evaporation of $10 \mathrm{~nm}$ of titanium followed by $100 \mathrm{~nm}$ of gold. No annealing step was necessary, either on Si or on InP, for the contacts to be ohmic.

Standard four-point probe $\mathrm{I}(\mathrm{V})$ measurements were carried out to evaluate current transport across the hetero-interface. Figure $1 \mathrm{~b}$ shows the $\mathrm{I}(\mathrm{V})$ curve for the $\mathrm{n}-\mathrm{InP} / \mathrm{n}-\mathrm{Si}$ isotype heterojunction. This curve is perfectly linear (ohmic) up to a current which corresponds to a current density of at least $50 \mathrm{~A} / \mathrm{cm}^{2}$ (the value assuming ideal current spreading). This result closely resembles those obtained for GaAs/InP [21] and $\mathrm{GaAs} / \mathrm{Si}$ [11] diodes fabricated using oxide-free direct bonding. This result is obtained despite the barriers that are indicated by the simulations. This is attributed to the large doping on both sides of the interface, inducing tunneling. These preliminary results of electrical injection through a planar oxide-free interface [22] indicate that it is possible to electronically integrate $\mathrm{InP}$ and $\mathrm{Si}$ at current densities relevant to laser diodes, without extra assisting materials, potentially simplifying photonic integration schemes.

\subsection{Mechanical Characterization}

For mechanical characterization, a 400-nm-thick InP membrane has been bonded on an Si substrate.

The surface bonding energy of the InP membrane when oxide-free bonded to sub-lambda patterned silicon was measured using the method described in Reference [16]. In this method, instrumented nano-indentation is used to locally debond InP from $\mathrm{Si}$. The debonded membrane forms a blister around the indent. The surface bonding energy is related to the geometry of the blister (length and height) and to two mechanical parameters of the membrane: the Young modulus of the InP material and the membrane thickness.

In detail, instrumented nano-indentation was performed in a Nanohardness tester from CSM Switzerland, equipped with a Berkovitch tip. The calibration procedure of Oliver and Pharr was used to correct for the load-frame compliance of the apparatus and the imperfections of the shape of the indenter tip. Loads between $5 \mathrm{mN}$ and $10 \mathrm{mN}$ were applied to the InP membrane. A Nomarski image of the blisters is shown in Figure 2a. The height and lateral extent of the blister were measured from atomic force microscopy (AFM) images of the blisters, acquired using a Veeco Dimension V AFM. An example of blister formed for an indentation load of $20 \mathrm{mN}$ is also shown in Figure 2a.

Cross-sections of the blister were subsequently prepared using focused ion beam etching and thinning. The cross-sections were observed in a JEOL 2200FS aberration-corrected scanning transmission electron microscope. An example of a cross-section prepared from an indent of InP bonded 
to patterned $\mathrm{Si}$ is shown in Figure 2b. The blister height and debonding crack length, visible in this image, are in excellent agreement with measurements from AFM. These measurements yielded a surface bonding energy of $1.07 \mathrm{~J} / \mathrm{m}^{2}$ in the case of InP bonded to bare Si and $0.548 \mathrm{~J} / \mathrm{m}^{2}$ in the case of InP bonded to patterned $\mathrm{Si}$. The ratio 1:2 between the two is in reasonable agreement with the filling factor of the patterns (Figure 2b-top), rather below 0.7 .

(a)

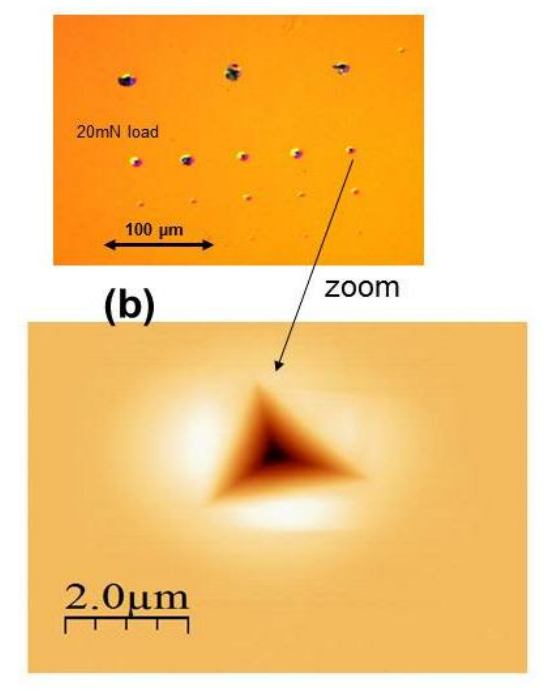

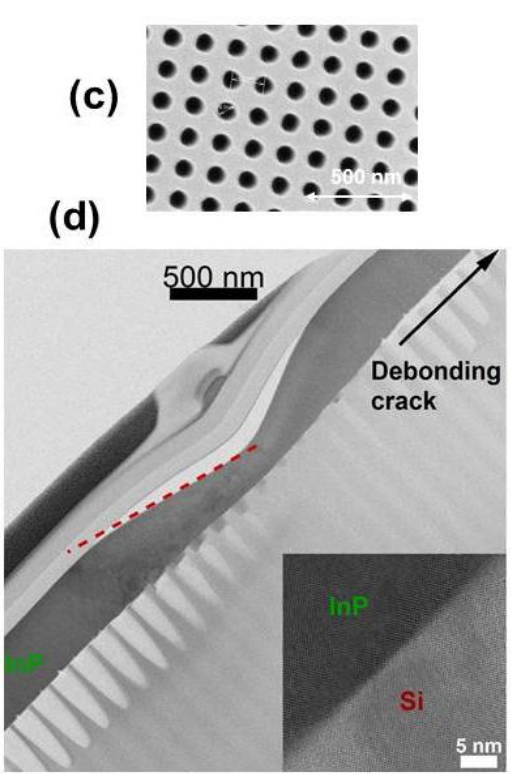

Figure 2. (a) Optical Microscope observation of the surface after indenting operation, (b) atomic force microscopy (AFM) image of a blister, (c) two dimensional Photonic Crystal (2D-PC) array of holes, (d) Cross-sections of an indent observed by TEM. Inset shows the $\mathrm{InP} / \mathrm{Si}$ interface far from the indent.

\subsection{Discussion}

Oxide-free bonding has been demonstrated to produce a hybrid interface without any defect at the atomic scale, thus offering the potential for any electrical/optical hybrid complex design and operation within a hybrid device. The main drawback limitation could come from the high processing temperature, which could be reduced down to the $400{ }^{\circ} \mathrm{C}$ range, but not lower than the congruence temperature of InP which is reported to be $376^{\circ} \mathrm{C}[23]$.

To perform bonding at CMOS compatible temperature, in the $250-300{ }^{\circ} \mathrm{C}$ range, the oxide-mediated bonding should then be considered. The scope, to retain most of the oxide-free bonded advantages, would be to reduce the thickness of the intermediate layer at its ultimate value, which is the single monolayer. Recent results point out that successful InP/Si bonding incorporating an $\mathrm{SiO}_{2}$ intermediate bonding layer thinner than $5 \mathrm{~nm}$ deposited by Atomic Layer Deposition (ALD) has been obtained [24]. The recovery of electrical conduction when diminishing this bonding layer thickness down to single a $\mathrm{SiO}_{2}$ monolayer will be interesting to investigate and analyze. 


\section{Hybrid InP on SOI Waveguide Including a Bi-Periodic Nano Structuration for Wavelength-Selective Transmission}

For an optical mode to be channeled in an SOI-based InP/Si hybrid structure, a lateral confinement has to be defined. This lateral confinement is usually defined in the Si guiding layer of the SOI stack since the major part of the optical mode is confined in this layer. Any classical rib or ridge design usually involves the ribs being far apart and will thus lead to adverse mechanical effects in the large unsupported areas when attempting oxide-free bonding onto the sole ribs. Hence we look for some flavor of planarized lateral cladding. We propose in this spirit to implement a nanostructuration for the transverse confinement. The optical waveguide has a generic shallow ridge shape, its core is not structured but both lateral claddings are composed of the same two dimensional Photonic Crystal (2D PC) operating essentially below its photonic gap, and offering mechanical support missing in the basic rib scheme. When properly tailored, thanks to the huge range of effective indices that can be obtained in silicon/air composites, such a nanostructure can provide virtually any spatial or spectral characteristics of the optical mode. The nanostructuration will preferably be a sub-wavelength, below band-gap periodicity in order to be compatible with a broad wavelength range between the long-wavelength photonic gap and the other short-wavelength optical window limits (multimodal onset, silicon or III-V absorption, etc.).

Here we demonstrate on a hybrid InP-on-SOI optical waveguide that a bi-periodic nanostructuration provides a wavelength selective transmission. The bi-periodicity is obtained by enlarging one hole each other longitudinal period. Hence, the short hole-to-hole distance is of sub-wavelength nature, whereas the double periodicity due to every other larger hole in the propagation direction fulfills the wavelength selective photonic role. The difference in hole sizes remains modest, hence the mechanical support issues are nearly unaffected by this choice, all the more if only a few rows (in the following only one row) is concerned.

\subsection{Design and Simulation}

For modal simulation, being in the sub-photonic band gap regime, Effective Medium Theory (EMT) has been implemented to represent the nanopatterned material as an effective cladding. On this basis, a 2D Modal analysis was performed in the plane transverse to the guide axis, providing the effective index of the guide fundamental mode, without longitudinal feedback. EMT is of interest since simulation of the exact geometry requires a tiny meshing of up to tens of holes on each side of the waveguide, which leads to highly demanding computational resources. Implementing EMT allows fast and quite accurate modal calculation, while investigating a large variation range for the several geometrical parameters. Then a 3D-FDTD is performed to determine the diameter of the enlarged holes that provide the wavelength selective feedback.

Specifically, the EMT is implemented to represent the 2D-PC material on the lateral cladding of the waveguide in the $x, y$ plane (Figure 3a), propagation being along the $\mathrm{z}$ direction. EMT has been demonstrated to fully represent a periodic nanostructured medium when its pitch $\Lambda$ is much smaller than the wavelength $\lambda, \alpha=\Lambda / \lambda<<1$ [25-27]. The 2D-PC is represented by a uniaxial material whose both ordinary and extraordinary indices are related to the hole diameter through the air filling fraction $f$ [28]. 
The effective modal index of the fundamental guided mode is then calculated with the commercial COMSOL RF mode solver module. We consider here a 550-nm-thick Si guiding layer-based design, which is large enough to get rid of the need of a 3D taper for further implementation inside a hybrid laser architecture. The selected geometrical parameters $(w, H, f)$ (see Figure 3 ) are those providing a large rejection against higher-order-modes in TE polarization. For this modal selection, we have followed the approach and criteria proposed by A. Pogossian et al. [29].

When the effective index of the fundamental TE mode is obtained, the added super-periodicity pitch $\Lambda_{\mathrm{DFB}}$ is calculated in order to provide wavelength selectivity at $1.55 \mu \mathrm{m}$. The $2 \mathrm{D}-\mathrm{PC}$ period in the propagation direction is then obtained, being half the super-period: $\Lambda_{\mathrm{EMT}}=\Lambda_{\mathrm{DFB}} / 2$, which is adequately in the sub-wavelength regime. Once the 2D PC longitudinal periodicity of typically $120 \mathrm{~nm}$ is determined from this simplified model, the 2D-PC transverse periodicity is kept to the subwavelength but slightly larger value of $150 \mathrm{~nm}$. The final 2D-PC thus becomes a rectangular array and the ordinary index of the EMT is then adjusted taking into account the rectangular geometry of the actual 2D-PC. The modal index is also re-calculated in order to finally adjust the periodicity in the propagation direction. The typical correction is a few $\mathrm{nm}$.

The geometry of the bi-periodic array being fixed, the abovementioned 3D-FDTD simulation is performed using the LUMERICAL commercial software to determine the larger hole's size to be chosen for the desired strength of wavelength selection, benchmarked here on a $80 \Lambda_{\text {EMT }}(\sim 10 \mu \mathrm{m})$ long device model. A $30 \%$ increase versus the nominal hole diameter has been found to produce a $5 \mathrm{~dB}$ transmission reduction at the stopband center.

(a)

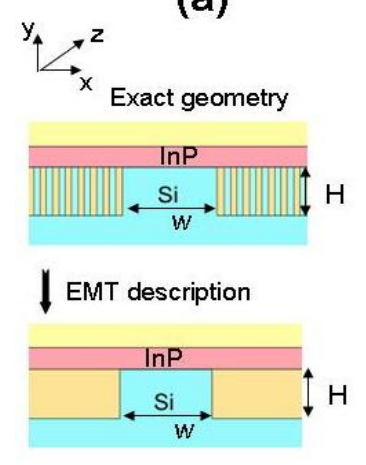

(b)

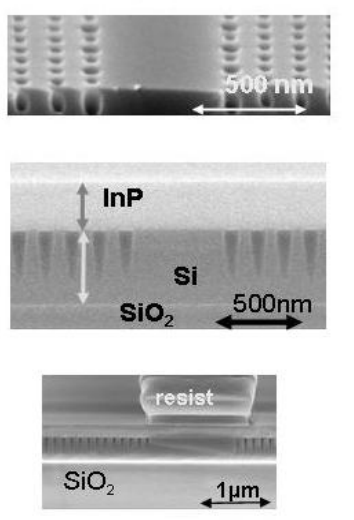

(c)

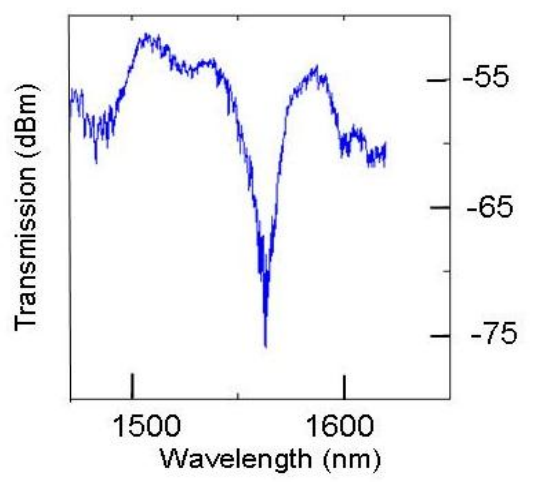

Figure 3. (a) Effective Medium Theory (EMT) representation of the 2D-PC used on the lateral cladding of the silicon waveguide, (b) SEM pictures: Top the silicon waveguide including the bi-periodic geometry Middle: the cleaved facet of the hybrid InP/Si waveguide Bottom: the resist line aligned on top to materialize the waveguide centre, (c) Spectrally resolved transmission of the hybrid waveguide.

\subsection{Fabrication and Measurement}

Such a bi-periodic waveguide is fabricated in the $550 \mathrm{~nm}$ thick silicon guiding layer of an SOI wafer kindly provided by SOITEC. The BOX layer thickness is $2 \mu \mathrm{m}$. The holes structuration is obtained by 
E-beam lithography and ICP dry-etching down to a typical depth of $250 \mathrm{~nm}$. Figure $3 \mathrm{~b}$ Top shows the bi-periodic nanostructured waveguide etched in silicon before bonding the III-V stack on it. Bonding is then performed in oxide-free form as described in [15]. After InP substrate removal, cleaved-facet waveguides are produced, a cleaved facet being shown on Figure $3 \mathrm{~b}$ (middle panel). For alignment purpose in the end-fire measurement, a resist line is defined as a marker on top of the waveguide center (Figure 3b bottom) as the waveguide is no more visible with silicon-based camera after bonding.

Transmission measurements are performed on an end-fire set-up including polarization maintaining tunable sources and injection fiber for TE polarization. Figure $3 \mathrm{c}$ shows the transmission in the case of a $0.6-\mu \mathrm{m}$-wide waveguide, with the stopband at $1.56 \mu \mathrm{m}$ evidencing the feedback behavior produced by the added periodicity, here on the first row of the 2D-PC. The stopband wavelength position is close to the $1.55 \mu \mathrm{m}$ expected one. The feedback efficiency (coupling strength related to the proper Fourier component of the effective index modulation) is large, producing a relatively broad dip, deeper than simulated, and spreading over $5 \mathrm{~nm}$ at half-peak height. This could be related to the impact of the larger holes being deeper than the others and deeper than simulated due to lag effect. During etching, a larger hole tends to be substantially deeper than nominal holes. These preliminary results [30] have to be consolidated before implementation in a DFB laser design.

\section{Conclusion}

This work takes place in the continuity of previous work on heteroepitaxial bonding of III-V materials on Silicon and on patterned Silicon-On-Insulator, whereby in-depth structural and optical investigations had pointed out the good features of our oxide-free bonding technique.

Here, we have shown a milestone toward a genuine optoelectronic integrated hybrid device. Concerning the hybrid interface itself, we have evidenced its ohmic electrical properties in the isotype case. Moreover, we have assessed the satisfactory mechanical strength of the interface for nanopatterned Si surfaces through precise bonding energy measurements. Concerning the optical architecture the sub- $\lambda$ nanostructuration is shown to be able, when including a tailored superperiodic feature, to produce simultaneously lateral guidance confinement as well as spectral feedback selectivity of the resulting guided mode. Patterned guiding SOI layers have been efficiently bonded to III-V materials under the oxide-free bonding procedure.

These elements are highly favorable to envisioning a hybrid laser with electrical injection across the hybrid interface, which will be beneficial for the device thermal characteristics. The pending issues along this road should address the choice of the p-n junction location, either within the III-V stack or at the hybrid interface (then a heterotype junction).

\section{Author Contributions}

K. Pantzas and A. Talneau conceived and designed the experiments, I. Sagnes delivered the MOVPE-grown III-V material, A. Itawi, K. Pantzas, E. LeBourhis, H. Benisty and A. Talneau performed the experiments, K. Pantzas, G. Patriarche, E. LeBourhis, A. Lupu, H. Benisty and A. Talneau contributed to the data exploitation; A. Talneau wrote the paper. 


\section{Conflicts of Interest}

The authors declare no conflict of interest.

\section{References}

1. Stadler, B.J.H.; Mizumoto, T. Integrated Magneto-Optical Materials and Isolators: A Review. IEEE Photon. J. 2014, 6, doi: 10.1109/JPHOT.2013.2293618.

2. Pantzas, K.; Patriarche, G.; Talneau, A.; Youssef, J.B. Bonding mechanism of a yttrium iron garnet film on $\mathrm{Si}$ without the use of an intermediate layer. Appl. Phys. Lett. 2014, 105, doi:10.1063/1.4896978.

3. Hulme, J.C.; Doylend, J.K.; Bowers, J.E. Widely tunable Vernier ring laser on hybrid silicon. Opt. Express 2013, 21, 19718-19722.

4. Fu, X.; Cheng, J.; Huang, Q.; Hu, Y.; Xie, W.; Tassaert, M.; Verbist, J.; Ma, K.; Chen, K.; Zhang, C.; et al. $5 \times 20 \mathrm{~Gb} / \mathrm{s}$ heterogeneously integrated III-V on silicon electro-absorption modulator array with arrayed waveguide grating multiplexer. Opt. Express 2015, 23, 18686-18693.

5. Stankovic, S.; Jones, R.; Sysak, M.S.; Heck, J.M.; Roelkens, G.; VanThourhout, D. 1310-nm Hybrid III-V/Si Fabry-Pérot Laser Based on Adhesive Bonding. IEEE Photon. Technol. Lett. 2011, 23, 1781-1783.

6. Lamponi, M.; Keyvaninia, S.; Jany, C.; Poingt, F.; Lelarge, F.; de Valicourt, G.; Roelkens, G.; van Thourhout, D.; Messaoudene, S.; Fedeli, J.; et al. Low-Threshold Heterogeneously Integrated InP/SOI Lasers With a Double Adiabatic Taper Coupler. IEEE Photon. Technol. Lett. 2012, 24, 76-78.

7. Ferrier, L.; Romeo, P.R.; Letartre, X.; Drouard, E.; Viktorovitch, P. 3D integration of photonic crystal devices: Vertical coupling with a silicon waveguide. Opt. Express 2010, 18, 16162-16174.

8. Fang, A.W.; Park, H.; Kuo, Y.; Jones, R.; Cohen, O.; Liang, D.; Raday, O.; Sysak, M.N.; Paniccia, M.J.; Bowers, J.E. Hybrid silicon evanescent devices. Mater. Today 2007, 10, $28-35$.

9. Itawi, A.; Pantzas, K.; Sagnes, I.; Patriarche, G.; Talneau, A. Void-free direct bonding of InP to Si: Advantages of low H-content and ozone activation. J. Vac. Sci. Technol. B 2014, 32, doi:10.1116/1.4863317.

10. Tanabe, K.; Iwamoto, S.; Arakawa, Y. Novel III-V/Si hybrid laser structures with current injection across conductive wafer-bonded heterointerfaces: A proposal and analysis. IEICE Elec. Ex. 2011, 8, 596-603.

11. Tanabe, K.; Watanabe, K.; Arakawa, Y. III-V/Si hybrid photonic devices by direct fusion bonding. Scient. Rep. 2012, 2, doi:10.1038/srep00349.

12. Fang, A.W.; Lively, E.; Kuo, Y.; Liang, D.; Bowers, J.E. A distributed feedback silicon evanescent laser. Opt. Express 2008, 16, 4413-4419.

13. Inoue, D.; Lee, J.; Hiratani, T.; Atsuji, Y.; Amemiya, T.; Nishiyama, N.; Arai, S. Sub-milliampere threshold operation of butt-jointed built-in membrane DFB laser bonded on Si substrate. Opt. Express 2015, 23, 7771-7778.

14. Patriarche, G.; Jeannès, F.; Oudar, J.L.; Glas, F. Structure of the GaAs/InP interface obtained by direct wafer bonding optimised for surface emitting optical devices. J. Appl. Phys. 1997, 82, 4892-4903. 
15. Talneau, A.; Roblin, C.; Itawi, A.; Mauguin, O.; Largeau, L.; Beaudoin, G.; Sagnes, I.; Patriarche, G.; Pang, C.; Benisty, H. Atomic-plane-thick reconstruction across the interface during heteroepitaxial bonding of InP-clad quantum wells on silicon. Appl. Phys. Lett. 2013, 102, doi:10.1063/1.4807890.

16. Pantzas, K.; Patriarche, G.; le Bourhis, E.; Troadec, D.; Itawi, A.; Beaudoin, G.; Sagnes, I.; Talneau, A. Evaluation of the surface bonding energy of an InP membrane bonded oxide-free to Si using instrumented nanoindentation. Appl. Phys. Lett. 2013, 103, doi:10.1063/1.4817675.

17. Tong, Q.-Y.; Gösele, U. Semiconductor Wafer Bonding; Wiley: New York, NY, USA, 1998.

18. Trucks, G.W.; Raghavachari, K.; Higashi, G.S.; Chabal, Y.J. Mechanism of HF etching of silicon surfaces: A theoretical understanding of hydrogen passivation. Phys. Rev. Lett. 1990, 65, 504-507.

19. Karim, A.; Abraham, P.; Lofgreen, D.; Chiu, Y.-J.; Piprek, J.; Bowers, J. Wafer bonded $1.55 \mu \mathrm{m}$ vertical-cavity lasers with continuous-wave operation up to $105{ }^{\circ} \mathrm{C}$. Appl. Phys. Lett. 2001, 78, 2632-2634.

20. Sze, S.M.; Ng, K.K. Physics of Semiconductor Devices, 3rd ed.; Wiley: New York, NY, USA, 2007; pp. 124-128.

21. Nakayama, K.; Tanabe, K.; Atwater, A.H. Improved electrical properties of wafer-bonded p-GaAs/n-InP interfaces with sulfide passivation. J. Appl. Phys. 2008, 103, doi:10.1063/1.2912717.

22. Pantzas, K.; Itawi, A.; Couraud, L.; Esnault, J.C.; le Bourhis, E.; Patriarche, G.; Beaudoin, G.; Sagnes, I.; Streque, J.; Talneau, A. Electrical transport across the heterointerface of InP membranes bonded oxide-free on Si. In Proceedings of the 26th International Conference on Indium Phosphide and Related Materials (IPRM), Montpellier, France, 11-15 May 2014.

23. Bentell, J.; Wennekes, F.; Salomonsson, F.; Hammar, M.; Streubel, K. Characterisation of n-InP/n-GaAs Wafer Fused Heterojunctions. Phys. Scripta. 1999, 79, 206-208.

24. Talneau, A.; Pantzas, K.; Durnez, A.; Patriarche, G.; Alamarguy, D.; le Bourhis, E. SiO $2 \mathrm{ALD}$ layers activation evidenced through III-V on Si hybrid bonded interfaces. In Proceedings of the 15 th International Conference on Atomic Layer Deposition (ALD), Portland, OR, USA, 28 June 2015.

25. Lalanne, P. Effective medium theory applied to photonic crystals composed of cubic or square cylinders. Appl. Opt. 1996, 35, 5369-5380.

26. Chen, F.T.; Craighead, H.G. Diffractive phase elements based on two-dimensional artificial dielectrics. Opt. Lett. 1995, 20, 121-123.

27. Brauer, R.; Bryngdahl, O. Design of antireflection gratings with approximate and rigorous methods. Appl. Opt. 1994, 33, 7875-7882.

28. Pang, C.; Benisty, H.; Besbes, M.; Pommarede, X.; Talneau, A. Oxide-free InP-on-Silicon-on-Insulator nanopatterned waveguides: Propagation losses assessment through end-fire and internal probe measurements. J. Lightwave. Technol. 2014, 32, 1048-1053.

29. Pogossian, S.P.; Vescan, L.; Vonsovici, A. The Single-Mode Condition for Semiconductor Rib Waveguides with Large Cross Section. J. Lightwave Technol. 1998, 16, 1851-1853. 
30. Pommarede, X.; Talneau, A.; Lupu, A.; Benisty, H. Wavelength-Selective Nanopatterned III-V on Si Hybrid Photonic Waveguide. In Proceedings of the 26th International Conference on Indium Phosphide and Related Materials (IPRM), Montpellier, France, 11-15 May 2014.

(C) 2015 by the authors; licensee MDPI, Basel, Switzerland. This article is an open access article distributed under the terms and conditions of the Creative Commons Attribution license (http://creativecommons.org/licenses/by/4.0/). 